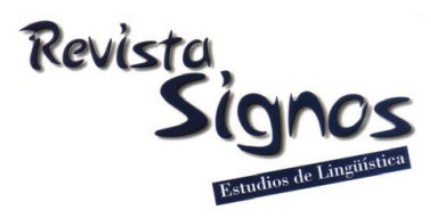

\title{
El informe de arbitraje científico: Sobre cortesía atenuadora y sexo del evaluador ${ }^{1}$
}

\author{
The scientific referee report: On attenuating courtesy and sex of \\ the evaluator
}

\author{
Julia Sanmartín Sáez \\ UNIVERSITAT DE VALÈNCIA \\ ESPAÑA \\ julia.sanmartin@uv.es
}

Recibido: 24-II-2020 / Aceptado: 14-VIII-2020

DOI: $10.4067 /$ S0718-09342020000300863

\section{Resumen}

Este artículo define, en una parte inicial teórica, el informe de arbitraje científico como género discursivo, ubicado en una cadena de géneros y con dos destinatarios, para poder contextualizar por qué se activa una estrategia de cortesía atenuadora. En una segunda parte empírica, la investigación pretende, por un lado, determinar en un corpus de informes de arbitraje cuáles son los recursos concretos para formular lingüísticamente dicha estrategia; y, por otro, desde el marco de la pragmática sociocultural y desde una perspectiva variacionista, comprobar si el sexo de los evaluadores de estos informes es un parámetro que incide en el empleo de una de las estrategias, la impersonalidad. Los resultados obtenidos confirman que se contemplan cuatro estrategias minimizadoras y que el sexo de los evaluadores repercute en el uso de la estrategia de impersonalidad y en la configuración de un estilo evaluativo singularizado por la conjunción de recursos lingüísticos.

Palabras Clave: Cortesía atenuadora, informe de arbitraje científico, impersonalidad, género discursivo, pragmática sociocultural. 


\begin{abstract}
This article defines, from a theoretical perspective, the scientific arbitration report as discursive genre, located in a chain of genres with two recipients, the purpose being to contextualize why a mitigating courtesy strategy is activated. The objective of the research is twofold: firstly, it attempts to determine which are the specific resources to formulate linguistically the above mentioned strategy in a corpus of arbitration reports; secondly, it aims to unveil whether the sex of the reports' evaluators is a parameter that influences the impersonality strategy in order to minimize the presence of the subject of the statement. The method of analysis is based on sociocultural pragmatics and on a variationist perspective. Results confirm that four minimizing strategies are contemplated, and that the sex of the evaluators affects the use of an impersonality strategy and the configuration of an evaluative style, which is characterized by a conjunction of linguistic resources.
\end{abstract}

Key Words: Attenuating politeness, scientific referee report, impersonality, discursive genres, sociocultural pragmatics.

\title{
INTRODUCCIÓN
}

En el marco de los géneros científicos, se sitúa el denominado 'informe de 'arbitraje científico' de artículos de investigación, encargado por revistas especializadas que desean adscribirse al sistema de evaluación por pares y doble ciego. Si hasta la fecha era un tópico recurrente aludir a la falta de estudios lingüísticos sobre este género textual, en la actualidad ha despertado un notable interés desde el marco del análisis del discurso, tanto en el ámbito anglosajón como en el hispánico, en especial, en los contextos universitarios de Chile y Venezuela. Así, destaca el proyecto de investigación chileno, La interacción socio-discursiva en la construcción colectiva del conocimiento cientifico: la dinámica interna del proceso de evaluación por pares, que ha cristalizado con la publicación de diversos resultados. Sin embargo, queda por analizar la incidencia de diferentes factores en la redacción de este documento, entre otros, la posible repercusión del sexo de los evaluadores o de la tradición discursiva a la que se adscriben.

La presente investigación se plantea determinar cuáles son las principales estrategias de atenuación empleadas en un corpus de informes redactados en español, y comprobar si existe alguna correlación entre el empleo de una de esas estrategias de cortesía atenuadora y el sexo de los evaluadores.

Para ello, la investigación se articula en dos secciones. En una primera sección, a partir de la revisión crítica de los estudios ya existentes sobre los géneros discursivos y sobre este género en particular, se caracteriza el informe de arbitraje científico en su contexto comunicativo para poder entender y vincular los elementos situacionales con sus correspondientes rasgos lingüísticos y sus estrategias pragmáticas, en concreto, con la activación de la considerada como 'cortesía atenuadora' o 'minimizadora'. 
En una segunda sección, de carácter empírico y en el marco epistemológico de los estudios de pragmática sociocultural sobre cortesía, se procede a analizar un corpus de 151 informes, compilado para esta investigación, con dos objetivos y dos análisis distintos: un primer objetivo consiste en comprobar si las estrategias de cortesía atenuadora detectadas en otros estudios se activan también en este corpus de informes; un segundo objetivo pretende averiguar si existe alguna correlación entre el empleo de una de estas estrategias de cortesía atenuadora, la impersonalidad, y el sexo del evaluador en un subcorpus de 40 informes.

\section{Marco teórico}

\subsection{El informe de arbitraje como género discursivo: La cadena de géneros}

En el marco del análisis de géneros discursivos (Askehave \& Swales, 2001; Bathia, 2002; Parodi, 2009), se indica que el informe de evaluación forma parte un proceso institucionalizado y reconocido por la comunidad de especialistas (Bolívar, 2008; Bolívar Orellana, 2014; Sabaj, González, Varas \& Pina-Stranger, 2015; Samraj, 2016; Fuentes, 2018), constituido por el propio artículo de investigación, la petición de informe por parte del editor, la redacción de los informes por los evaluadores, las posibles modificaciones del investigador y el dictamen del editor. Todos estos documentos, con autonomía formal y funcional, aparecen como elementos de una 'cadena de géneros discursivos' (Bolívar, 2008; Suid, 2016; Suid, Sabaj \& González, 2018). Cada uno de estos géneros solo puede aparecer en un momento secuencial y cronológico, a diferencia de lo que acontece en un 'macrogénero', unidad caracterizada por servir de soporte a distintos géneros integrados en ella. Es más, el informe debe escribirse en un plazo temporal indicado por el editor de la revista. Así pues, se considera que este informe, como género discursivo, se inserta en la cadena de textos que integra el sistema de arbitraje, en inglés, peer-review referee system, ya que es un tipo de intervención reactiva a petición de un editor para que se evalúe un artículo de investigación.

Se describiría como un 'género reactivo' ya que es una reacción a la petición del editor (Suid, 2016), pero también responde y tiene en cuenta el propio artículo. El autor-investigador contestará a los comentarios realizados en el informe, a modo de alegaciones, y el editor de la revista enviará las modificaciones realizadas en el artículo a los evaluadores para que den el visto bueno para la publicación o, en algún caso, el editor asumirá el papel de juez que tiene en cuenta si se han efectuado o no los correspondientes cambios y, por consiguiente, decidirá publicar o no el artículo. De hecho, el informe de arbitraje se asemeja a un juicio: el léxico empleado (como 'veredicto') y todo el proceso implicado así lo confirman. 
Sabaj, González, Astudillo, Varas, Fuentes, Cabezas, Squadrito y Pina-Strangerl (2018) consideran todo este proceso de arbitraje como una práctica sociodiscursiva de carácter técnico, y evaluativo puesto que requiere del conocimiento científico, se evalúa y cristaliza en un discurso final: el artículo. Se describiría, pues, como un proceso discursivo complejo con una estructura polifónica, ya que el artículo final implica una diversidad de autores al tenerse en cuenta los comentarios del informe y tener que modificarse el artículo para su publicación. De ahí, la clasificación del informe como "género escrito interactivo de carácter privado y forma parte de una cadena de géneros que culmina con la publicación del artículo de investigación" (Suid et al., 2018: 1) o como "un género clave para explicar la forma en que se construye colectivamente el conocimiento científico" (Astudillo, Squadrito, Varas, González \& Sabaj, 2016: 20).

En esta investigación la atención se focaliza en el denominado por la comunidad de referencia con dos etiquetas, 'informe de evaluación'-más empleada en el español peninsular-o informe de arbitraje' de revistas científicas, que guarda una estrecha relación con todos aquellos textos que cumplen una función comunicativa similar: evaluar o enjuiciar las características de un fenómeno y, en consecuencia, aprobarlo o rechazarlo. En este sentido, enlazan con otros dictámenes también elaborados en el propio ámbito académico universitario, como aquellos que evalúan la capacidad investigadora de los sujetos, los sexenios de investigación, o aquellos que valoran la concesión de los proyectos de investigación. Todos ellos se podrían considerar como 'familias de géneros', ya que presentan una función similar y actúan en el mismo contexto profesional o comunidad discursiva: el ámbito científico-académico. Algunos estudios, como el de Bolívar Orellana (2014) o Fuentes (2018), lo consideran como un 'género académico evaluativo'. Sin embargo, aunque se trata de un género vinculado al ámbito universitario, se podría clasificar más propiamente como un género inserto en un circuito científico al desarrollar una evaluación de un artículo de una revista científica y, además, presenta una conexión con el ámbito editorial, ya que la petición del informe la solicita un sujeto con la función de editor de una revista.

\subsection{El juego de identidades: Anonimato, autoridad, asimetría y destinatarios}

En esta cadena de géneros, cabe aludir a un elemento esencial: el juego de identidades y la asimetría en la relación funcional (Austudillo et al., 2016). El editor o equipo editorial es el único que conoce la identidad del autor y de los revisores y es conocido por ellos. De hecho, actúa como mediador de información entre ambos para garantizar su anonimato. Ni autor ni revisores conocen sus respectivas identidades. Es un sistema de doble ciego por dos motivos: por un lado, para salvaguardar las imágenes, puesto que una valoración negativa puede dañar la imagen del autor (Kourilova, 1998) y, especialmente, la del evaluador (es el emisor de la afrenta); por 
otro, para garantizar la objetividad del evaluador y evitar posibles presiones (Bolívar Orellana, 2014).

Y, más allá de la protección del anonimato que salvaguarda la imagen de los sujetos (autor y evaluadores), cabe destacar la asimetría funcional que se establece: el editor decide en última instancia si se publica el artículo, los evaluadores emiten su juicio y se encuentran en una posición de superioridad respecto al autor, que debe acatar todas las decisiones si desea que su artículo sea publicado; así también lo afirman Suid et al. (2018: 3):

"En este sentido, los evaluadores se encuentran en una ventaja de poder respecto del autor, ya que tienen influencia en el destino de los manuscritos enviados a la revista."

En relación con la distancia social, no ha lugar su valoración como parámetro por el anonimato del evaluador. Dicho en otras palabras, en una interacción cara a cara se suma la relación funcional y la adscripción social, pero en este género discursivo, la segunda queda neutralizada. En todo caso, se valorará el perfil más o menos consolidado del evaluador como autoridad en la materia. El informe es redactado por, al menos, dos especialistas o expertos (o peer review) en el tema objeto de estudio, quienes evalúan el artículo, recomiendan o rechazan su publicación, e incorporan sugerencias que deben ser tenidas en cuenta por los autores de los artículos para poder publicar sus textos y, de este modo, los evaluadores se transforman en una especie de figura de autoridad. Es más, se reconoce explícitamente en las normas de la revista que los evaluadores serán expertos en la materia: a veces seleccionados a partir de la propia bibliografía del artículo.

En esta cadena de géneros, parece esencial considerar que el evaluador tiene presente que su informe se dirige a dos receptores en una especie de juego de destinatarios e identidades semiocultas: por un lado, el texto redactado por el evaluador es un informe, solicitado por el equipo editorial; por ello, el evaluador elabora un dictamen de evaluación para un 'destinatario directo': el editor (Varas, 2015). Por otro lado, el segundo receptor del informe es un 'destinatario indirecto', el propio autor, quien debe tener en cuenta las observaciones del evaluador para que se pueda publicar su artículo.

Por ello, numerosos informes constan de dos partes en los comentarios: una de ellas visible al autor y otra exclusiva para el editor de la revista, la cual no se enviará al autor. Esto confirma que, en definitiva, el editor de la revista es el destinatario primario.

En la Figura 1, se intenta reflejar el juego de identidades y emisores (EM.) /destinatarios o receptores (RE.), con las distintas relaciones de asimetría funcional ${ }^{2}$ (Fuentes, 2018) en las sucesivas fases de la cadena de géneros. El equipo editorial 
actúa como mediador y garante del anonimato, y como emisor o receptor en todos los eventos comunicativos:

\begin{tabular}{|c|}
\hline $\begin{array}{l}\text { EM: AUTOR (IDENTIFICADO) petición publicación de artículo (relación funcional -poder) } \\
\text { RE. EQUIPO EDITORIAL (IDENTIFICADO): aceptación o no evaluación artículo } \\
\text { (relación funcional + poder) }\end{array}$ \\
\hline CON IDENTIDAD (2) relación simétrica directa Editor / Evahıador \\
\hline EM. EQUIPO EDITORIAL (IDENTIFICADO) solicitud evaluación (relación funcional =poder) \\
\hline RE. EVALUADOR1 (IDENTIFICADO) aceptación evaluación (relación funcional =poder) \\
\hline RE. EVALUADOR 2 (IDENTIFICADO) aceptación evaluación (relación funcional =poder) \\
\hline CON ANONIMATO (3) relación asimétrica indirecta Evaluador / Autor \\
\hline EM. EVALUADOR1 (ANÓNIMO) emisión informe \\
\hline EM. EVALUADOR2 (ANÓNIMO) emisión informe \\
\hline RE. DIRIGIDA AL EQUIPO EDITORIAL (relación funcional =poder) \\
\hline RE. RECIBIDA POR EL AUTOR (ANÓNIMO) recepción informe (relación funcional -poder) \\
\hline
\end{tabular}

Figura 1. Identidad, anonimato y relaciones funcionales desiguales.

\subsection{La función evaluadora y los actos de habla (des)corteseses}

Con el proceso de revisión por pares y doble ciego, se pretende garantizar la calidad de la revista. Los evaluadores preservan su identidad y se mantienen en el anonimato ya que deben juzgar y evaluar la investigación elaborada por los colegas del ámbito.

De este modo, el informe se convierte en un texto anónimo y de carácter privado, confidencial, evaluativo y directivo (Bolívar Orellana, 2014); para Austudillo et al. (2016) destacaría también esa consideración de 'privado' y 'confidencial', lo que explicaría su falta de estudios. Es lo que Swales (1996) etiquetó como 'género oculto’.

Para reconocer el informe de evaluación o arbitraje como género discursivo es esencial, más que las pautas formales o el esquema organizativo que presente, su ubicación en la cadena de géneros y el tenor funcional o la función principal que asume (evaluar), ya que su macroestructura organizativa puede ser muy diversa, según establezcan los criterios de la revista, esto es, su organización estructural puede experimentar diversas formas, tal y como se constata en Bolívar (2008: 50) al revisar tres revistas distintas: "Cada informe responderá a expectativas creadas y exigidas por un grupo editorial particular."

Las revistas de investigación establecen diferentes directrices y modelos de evaluación, los cuales pueden cambiar con el paso del tiempo, como sucede en cualquier género discursivo (Bhatia, 2002), y, de este modo, condicionan el tipo de informe realizado. El informe que los evaluadores deben completar puede constar de varias partes: a) un texto sintético en el que asignan valores numéricos a unas preguntas formuladas, a modo de escalas de Lickert. (Austudillo et al., 2016); b) un texto de carácter libre, en el que se pueden redactar comentarios; c) la recomendación de publicar o no el texto: aceptado, aceptado con enmiendas menores, aceptado con 
enmiendas mayores, y rechazado. d) un comentario dirigido exclusivamente a la redacción de la revista que el investigador evaluado no recibirá.

En algunas revistas no solo se solicita al evaluador que lleve a cabo una valoración, sino también una propuesta que permita de algún modo mejorar el artículo: así, por ejemplo, se destaca en el análisis llevado a cabo por Bolívar Orellana (2014) en relación con la revista Paradigma; y, por ello, se consideraría como una segunda función secundaria.

Autores, como Gosden (2003), Mungra y Weber (2010) y Bolívar Orellana (2014), han abordado la determinación de los aspectos más evaluados y los criterios utilizados en la valoración: contenido y tratamiento adecuado de los referentes teóricos; existencia de rigor metodológico; y la correcta escritura o seguimiento de las pautas estructurales del género artículo de investigación.

En relación con el tenor comunicativo de la evaluación, se constata una serie de 'actos de habla' (con propósitos comunicativos), actos que se describen e ilustran con un ejemplo procedente del corpus de informes que después se indicará en 2.2: unos de carácter cortés, como la valoración positiva o alabanza, en (1); o la valoración neutra, al realizar una mera descripción (quizá los menos frecuentes porque no tiene sentido la mera descripción sin un valor evaluativo), en (2); otros de carácter (des)cortés, como es la valoración negativa o crítica: describen errores o lo que falta, en (3); el mandato de modificación para mejorar el texto y que sea publicado, como añadir algo o rectificar, en (4); la sugerencia de modificación en (5), si bien las sugerencias deben interpretarse como imposiciones si el autor quiere publicar su artículo:

(1) Es un trabajo riguroso, bien planteado y bien organizado, muestra conocimiento de la materia y de la bibliografía pertinente.

(2) La investigación aplica los postulados de la pragmática sociocultural y de la teoría de la descortesía verbal de enfoque social y discursivo al análisis de un corpus digital.

(3) Creo que la temporalización es demasiado vaga, tanto en su totalidad como en el tiempo de cada actividad, que no se indica

(4) Evitar la personalización del texto: el uso del yo o del nosotros está fuera de lugar en cualquier texto científico.

(5) Se recomiendan los cambios propuestos en el mismo artículo y una revisión en profundidad de todo el texto, atendiendo a dichas normas.

Según Fortanet (2008), las críticas son los tipos de actos de habla más frecuentes. Para Sabaj et al. (2018) el tipo de acto realizado está vinculado con el veredicto del evaluador (aceptar, rechazar o condicionado). Estos autores, además, matizan que las críticas están motivadas también por la propia productividad de los evaluadores: 
“el evaluador sin productividad realiza una revisión más minuciosa por la necesidad de ir legitimándose en un campo disciplinar, en este caso, mostrando ante el editor que es capaz de realizar una evaluación detallada y exhaustiva." (Sabaj et al., 2018: 21)

\subsection{La cortesía atenuadora como estrategia pramalingüística}

El informe de evaluación no pretende ni transgredir las normas de cortesía ni dañar la imagen de los autores del artículo juzgado ni la suya propia. Por ello, en principio, los evaluadores se valen de distintas estrategias para atenuar sus aserciones, en especial, cuando su valoración es negativa, o en sus imposiciones; de ahí, la ‘cortesía atenuadora' o 'mitigadora’. En esta línea, Bolívar (2008: 61) apunta:

"El hecho de que los árbitros traten de mitigar sus evaluaciones cuando dictaminan modificaciones mayores, nos indica un deseo de respetar al otro, su imagen, su prestigio profesional. La cortesía, por lo tanto, tiene en el arbitraje un papel muy importante, que amerita un mayor estudio."

Este concepto de 'cortesía mitigadora' se enmarca en los estudios sobre (des)cortesía verbal (Brown \& Levinson, 1987; Haverkate, 1994), especialmente, en el marco de la pragmática sociocultural (Bravo \& Briz, 2004; Albelda \& Barros, 2013), y constituye parte del marco teórico desde el que se aborda el objeto de análisis. Así, Brown y Levinson (1987) incorporan las nociones de 'imagen' y 'territorio' del sociólogo Gofffman, y diferencian entre 'imagen negativa' (o territorio), el deseo del sujeto de libertad de actuación, sin imposiciones; y la 'imagen positiva' o la voluntad de aprobación o valoración por parte de otros sujetos.

En el informe de arbitraje la imagen del evaluador está protegida por el anonimato frente al autor, pero está en exposición frente al equipo editorial que podría valorar su informe como descortés. De este modo, se produce un juego sutil de identidades y afectaciones de las imágenes. El evaluador, protegido por el anonimato, debe salvaguardar su imagen respecto al editor, que también leerá su informe y podrá juzgar si respeta las normas de cortesía académica (Suid et al., 2018).

Véase como muestra del carácter privado, de la necesidad de valoración objetiva y del mantenimiento del anonimato del evaluador, la siguiente carta del ejemplo (6), elaborada por una revista, en la que se insiste en expresar de forma neutra las valoraciones negativas:

(6) Adjunto le envío dicho informe y el artículo para evaluar [.... . Cabe decir que el autor recibirá el informe tal cual lo redacte, por lo que le rogamos que intente expresar sus opiniones de la forma más neutra posible en el caso de una evaluación negativa. Por otro lado, le informamos que los nombres de los revisores no son comunicados al autor [...] Por último, si usted cree que puede conocer al autor y considera que no podría realizar una valoración objetiva a este respecto, recomendamos a los revisores que nos lo comuniquen. 
Curiosamente, según lo apuntado en estudios anteriores (Bolívar, 2011), predominan los comentarios negativos; y de ahí la consideración del evaluador como corrector: "el papel que suele asumir el evaluador de un artículo científico es el de un corrector que señala los errores del texto que evalúa” (Austudillo et al., 2016: 125).

Ahora bien, esta cortesía atenuadora podría verse afectada, al menos, por cinco factores: el sexo, la consolidación académica, la adscripción geolectal/cultural de los evaluadores e, incluso, si el juicio de valor resulta favorable o no y el tipo y/o temática de la revista. Cabría obtener un corpus representativo de informes en los que se pudiera comprobar cómo inciden estos factores.

Por otro lado, como ha estudiado Paltridge (2015), el peligro de la atenuación radica en que puede que no sea interpretada correctamente por los investigadores jóvenes y sin experiencia: estos pueden considerar que la propuesta de modificación del artículo presentada por los evaluadores es opcional.

\section{Marco metodológico}

\subsection{Objetivos e hipótesis}

Esta investigación pretende averiguar, por un lado, si en un corpus de informes de distintas revistas se activan estrategias de cortesía atenuadora y cuáles son; y, por otro, si existe una correlación entre el sexo de los evaluadores y el empleo de una de estas estrategias: la impersonalidad. En concreto, se toma como objeto de estudio cómo se minimiza el sujeto del enunciado cuando coincide con la figura del evaluador o del autor, es decir, cómo el evaluador, varón o mujer, se presenta discursivamente y se refiere al autor del artículo, ya que se parte de la hipótesis de que la variable sexo puede afectar al modo en que los evaluadores emplean las diferentes estrategias de cortesía al difuminar su presencia como fuente de la crítica negativa o de la imposición al otro.

Estos objetivos pretenden responder, pues, a dos preguntas: 1- ¿Cuáles son los mecanismos lingüísticos para atenuar las críticas que se formulan en los informes de arbitraje? 2- ¿Existe alguna correlación entre la minimización del sujeto del enunciado y el sexo de los evaluadores?

\subsection{Descripción del corpus de informes de arbitraje}

Para determinar las distintas estrategias de cortesía atenuadora empleadas en los informes de arbitraje, se ha obtenido un corpus de 151 informes, a pesar de los escollos metodológicos que supone su compilación: por su carácter privado (Bolívar, 2008), por la consideración de la confidencialidad que se establece al formar parte de 
un proceso evaluador y por el posible daño que se puede derivar al exponer la imagen del evaluado y también del evaluador.

Quizá esto explica la dificultad que se ha tenido que soslayar para la recopilación de las muestras así como lo reducido del corpus de estudios similares: por ejemplo, en Bolívar (2008) se describen 25 informes de tres revistas; en Bolívar Orellana (2014) se analizan 24 informes de arbitraje de la revista Paradigma; Austudillo et al. (2016), 56 informes de la revista Onomázein; tan solo se amplían en Sabaj et al. (2018) a 318 informes y, en especial, se analizan 118 de tres revistas distintas, al tratarse de un proyecto de investigación.

En esta investigación, se han obtenido 151 informes de 27 revistas, agrupados en dos tipos:

a) 31 informes: 10 informes de 10 evaluadores anónimos y 21 informes de 4 evaluadores identificados, de 26 revistas distintas: Spanish in context, IPRA.Pragmatics 18, Anuario de Filología, Revista Normas, Discourse Studies, Revista Signos, Sintagma, Lengua y migración, ELUA, Borealis, EDICE, Lingüística en la Red, Onomázein, etc.

b) 120 informes de 120 evaluadores identificados por sexo de una única revista, Revista Foro de Profesores de Español como Lengua Extranjera, de 2017, 2018 y 2019. Esta revista, publicada por la Universidad de Valencia, está indexada desde 2013 en diversas plataformas y su objetivo es proporcionar material teórico y didáctico a los profesores del ámbito de la enseñanza y aprendizaje de español como lengua extranjera. Se seleccionan artículos que superan los índices de calidad de la revista y la revisión por pares ciegos.

\subsection{Análisis descriptivo: La delimitación de las estrategias atenuadoras en el corpus}

\subsubsection{Diseño del análisis descriptivo}

Para la caracterización pragmalingüística del corpus, se han considerado diferentes elementos que operan para construir esa mitigación, tomados de la ficha de atenuación, propuesta por Albelda, Briz, Cestero, Kotwica y Villalba (2014) y de los aspectos ya valorados en el estudio de Suid et al. (2018), si bien se han unificado en cuatro apartados. Si se consideran como elementos insertos en el marco de la 'cortesía atenuadora' es porque tratan de minimizar las críticas o las imposiciones de modificación del artículo, tal y como se observa en el ejemplo (7):

(7) En general, el artículo es interesante, pero debe ser revisado, se le deben aplicar los cambios que se sugieren para poder ser publicado en una revista científica como Foro ELE. 
En este caso, la cortesía atenuadora tiene lugar para autoproteger la imagen del evaluador frente al editor y reparar la amenaza a la imagen del autor frente al editor ${ }^{3}$ : se alaba primero ('interesante') para contrarrestar la imposición al autor ('debe revisar'). Es esencial que el editor comparta la evaluación realizada y, por ello, cabe justificar las decisiones, y que el autor comprenda las observaciones (Bolívar Orellana, 2014).

Se han diferenciado cuatro tipos de estrategias de cortesía minimizadora en el corpus de 151 informes: a) posibilidad y condicional, b) subjetividad y recomendación, c) estructuración informativa, d) impersonalidad.

\subsubsection{Resultados del análisis descriptivo}

Las cuatro estrategias establecidas aparecen de un modo frecuente en el corpus, especialmente en los informes de evaluación publicables con revisión.

\section{a) La posibilidad y el condicional como estrategias para aminorar la fuerza ilocutiva}

En este apartado se integran tres aspectos recurrentes en el corpus: el uso del condicional, de la perífrasis de probabilidad y de los adverbios de duda.

En el ejemplo (8), se trata de una imposición de corrección atenuada por el modo condicional utilizado en 'debería' y 'convendría':

(8) A su vez, debería revisarse la redacción de algunos párrafos que resultan confusos [...] Convendría revisar la utilización de las comillas, ya que en alguna ocasión se han utilizado las comillas altas o inglesas, en lugar de las bajas o españolas.

Se observa también la utilización de estructuras condicionales como aparecen en el ejemplo de (9), sobre todo vinculadas a la perífrasis de posibilidad 'podría ser':

(9) (...) considero que podría ser mucho más interesante y tener más impacto, si (el artículo) presentara unas referencias bibliográficas más claras y contundentes sobre este tema.

Un estilo impositivo sin atenuación sería el del ejemplo (10), más habitual cuando se solicitan cambios formales o de expresión 4 :

(10) Para ser de interés el autor debe presentar unas referencias bibliográficas más claras y contundentes

Los otros dos recursos para aminorar es recurrir a perífrasis de posibilidad para reducir certeza ('podría reformularse') y utilizar locuciones adverbiales de duda ('quizá'), tal y como sucede en el ejemplo (11), en el que se emplean los tres recursos 
de un modo simultáneo (junto con una justificación argumentativa) como propuesta del evaluador para que el autor modifique su artículo:

(11) Quizá podría reformularse el fragmento reproducido ya que puede resultar ambiguo.

\section{b) La subjetividad y la recomendación como estrategias para aminorar la fuerza ilocutiva}

En los informes se constata el empleo de verbos de recomendación o sugerencia frente a la petición o mandato de una actuación concreta como una manera de atenuar la invasión en el territorio del otro: 'recomiendo' o 'sugiero', como ocurre en el ejemplo (12), en los que se suele detectar el recurso a la primera persona del singular:

(12) Dado que el trabajo es susceptible de ser leído y aplicado en diversos países con diferentes sistemas, recomiendo reformular el contexto de aprendizaje $(\ldots)$

En esta línea, Sabaj et al. (2018: 18) indican:

"Cuando los evaluadores recomiendan la publicación del trabajo, realizan más sugerencias, en cambio, si condicionan la publicación del trabajo, entonces directamente solicitan acciones."

En cualquier caso, tanto la recomendación como el condicional serían un acto de habla deferente, sujeto a una posible mala interpretación (Suid et al. 2018). De hecho, como sucede en el ejemplo (13), se indica de un modo explícito que las recomendaciones deben seguirse para poder publicar el texto:

(13) Proponemos el artículo XXX para su publicación, siempre y cuando el autor atienda a las sugerencias de cambio profundo que se anotan en comentarios al margen del texto y a continuación.

Además de la recomendación, en los informes se documentan performativos de creencia u opinión ${ }^{5}$, verbos doxásticos, como 'creo' o 'pienso' para expresar el foco de subjetividad y, por ello, reducir la asertividad en relación con lo dicho, especialmente de las críticas. Como muestran Bolívar (2008) y Suid et al. (2018), se interpreta como una estrategia para proteger la imagen del evaluador. Se trata de una protección, en especial, frente al editor, tal y como se constata en el ejemplo (14), comentario negativo:

(14) Creo que la temporalización es demasiado vaga, tanto en su totalidad como en el tiempo de cada actividad, que no se indica. 


\section{c) La estructuración informativa como estrategia atenuadora de compensación}

Los estructuradores y conectores de la información de tipo causal ('puesto que', 'pues') permiten incorporar las diversas argumentaciones como prueba de que lo expresado, la crítica, procede de un razonamiento, tal y como se ha ejemplificado anteriormente en (11). Sin embargo, quizá la estrategia más habitual es un movimiento contraargumentativo del tipo valoración positiva con 'pero', seguido de 'no obstante' o 'sin embargo'. Destacar lo positivo y después elaborar una crítica se convierten en una estrategia de cortesía atenuadora porque lo negativo queda compensado con lo positivo anterior, tal y como han indicado diferentes autores en relación con los informes (Kourilova, 1998; Bolívar, 2008; Bolívar Orellana, 2014; Samraj, 2016; Suid et al. 2018); es felicitar para criticar, como acontece en (15):

(15) A nivel de contenido, la unidad didáctica es correcta y está justificada su realización en los grupos definidos a lo largo del trabajo (...) Ahora bien, convendría realizar algunas precisiones.

\section{d) La impersonalidad como estrategia para aminorar la responsabilidad}

En la descripción de esta estrategia solo se han diferenciado tres manifestaciones del sujeto evaluador en el texto en actos de habla en los que sujeto verbal y emisor coinciden: (1) el 'se' impersonal (en, 'se considera que'), (2) el 'yo' evaluador (en, 'considero que') o (3) el 'nosotros' de modestia (en, 'consideramos que). De estas tres formas, la impersonal es la que funciona como un recurso para aminorar la responsabilidad con lo dicho.

En los ejemplos de la primera persona de singular, es habitual el tipo de verbo de cognición que favorece el uso de esa primera persona ('pienso'). En este caso, sería también una forma de atenuar desde la consideración de la subjetividad del 'yo' (equivalente a 'es solo mi opinión'), como ya se ha expuesto en el apartado b).

La impersonalidad también se contempla en relación con la figura del autor, destinatario indirecto del informe, en expresiones que implican cierto mandato: 'se podría' en lugar de 'el autor podría'. Es importante destacar que el destinatario de la evaluación suele aparecer omitido, esto es, no se encuentra una alusión directa al autor del texto. Para Bolívar Orellana (2014), es un modo de evaluar la investigación y no a su autor.

Así, en los informes se observa la omisión de apelación directa en actos de habla dirigidos al autor (como 'se deben corregir los siguientes errores') frente a la referencia explícita al autor ('el autor debe corregir los siguientes errores'). 


\subsection{Análisis de variación: La impersonalidad y el sexo de los evaluadores}

\subsubsection{Diseño del análisis de variación}

En este análisis se va a tener en cuenta si el sexo de los evaluadores determina el tipo de cortesía atenuadora empleada, parámetro ya considerado en otros géneros discursivos sobre atenuación y cortesía. Así, Cestero (2017) y Albelda (2018) constatan diferentes comportamientos discursivos de hombres y mujeres en entrevistas del Proyecto para el Estudio Sociolingüistico del Español de España y de América (PRESEEA): entre otros, los hombres recurren más a la impersonalidad que las mujeres. Por su parte, García Mouton (1999) alude a las distintas estrategias usadas en varios discursos: la mujer resulta una hablante más cooperativa y cortés.

Hasta la fecha, en los informes de evaluación solo se ha contemplado la incidencia del perfil académico de los evaluadores (Sabaj et. al, 2018; Suid et al., 2018) y, especialmente, el tipo de evaluación. Bolívar Orellana (2014) apuntó que la aceptación o rechazo de publicación repercute más directamente en la configuración lingǘstica del informe, incluida la cortesía, que la productividad investigadora de los evaluadores.

Así pues, este estudio se centra en un aspecto novedoso: comprobar cómo se presenta el evaluador y el autor del artículo como sujetos discursivos y su correlación con el sexo del evaluador. Del corpus general de 151 informes se ha seleccionado un subcorpus de 40 informes de la Revista Foro de Profesores de Español como Lengua Extranjera de 2017 y 2018 para poder llevar a cabo un estudio contrastivo de informes similares y con cierta homogeneidad estructural al formar parte de la evaluación de una única revista. De los 120 informes compilados de esta revista, se descartaron los 30 informes de 2019 al emplear un sistema de evaluación más sistemático que los años anteriores (con preguntas numéricas tipo test y con un doble apartado final de comentarios: unos reservados para el editor y otros remitidos al autor). De los informes de 2017 y 2018 se seleccionaron solo aquellos que presentaran una crítica o imposición de actuación, objeto de la cortesía atenuadora, frente a los meros informes de aceptación y valoración positiva. Tampoco se incluyeron ni los excesivamente breves ni con un estilo telegráfico. Finalmente, cabe precisar que el número de mujeres evaluadoras es más del doble que el de varones. Todos estos condicionamientos llevaron a la delimitación de un subcorpus de 40 informes, con un número idéntico de informes emitidos por varones y mujeres, en los que la publicación estaba sujeta a la inclusión de modificaciones (más o menos importantes): publicable con revisiones.

En estos informes, se ha tenido en cuenta el sujeto discursivo de los verbos, el cual se corresponde con la descripción de la impersonalidad como estrategia para aminorar la responsabilidad del apartado anterior. Esta selección ha soslayado el problema de la delimitación de la unidad 'comentario' de análisis previos (Sabaj et al., 2018), y permite 
cierta sistematicidad en el análisis al centrarse únicamente en la consideración de los sujetos verbales. Todo ello, por supuesto, se lleva a cabo en el cotexto correspondiente para poder evaluar las estrategias de cortesía atenuadora. En definitiva, se han observado dos cuestiones:

I) si el sujeto evaluador y enunciador aparecía:

(1) como 'se' impersonal sujeto verbal ('se observa')

(2) como una primera persona sujeto verbal

(2.1) el 'yo' evaluador ('observo')

(2.2) el 'nosotros' de modestia ('observamos')

II) si el autor y destinatario aparecía

(1) como (sujeto) impersonal en actos de habla dirigidos al autor ('se deben corregir los siguientes errores' o 'sería interesante corregir los siguientes errores')

(2) como sujeto verbal ('el autor debe corregir los siguientes errores')

(3) sustituido por el propio 'artículo', considerado como objeto de descripción crítica ('el artículo presenta los siguientes errores').

\subsubsection{Resultados del análisis de variación: la correlación entre sexo e impersonalidad}

Los resultados cuantitativos han sido los siguientes, tal y como se muestra en la Gráfico 1, teniendo en cuenta que se cuantifica como 'primera persona' la aparición de formas de singular 'yo' y/o plural 'nosotros' en un mismo informe, y que se ha considerado que un fenómeno aparecía en un informe con independencia de su recurrencia:

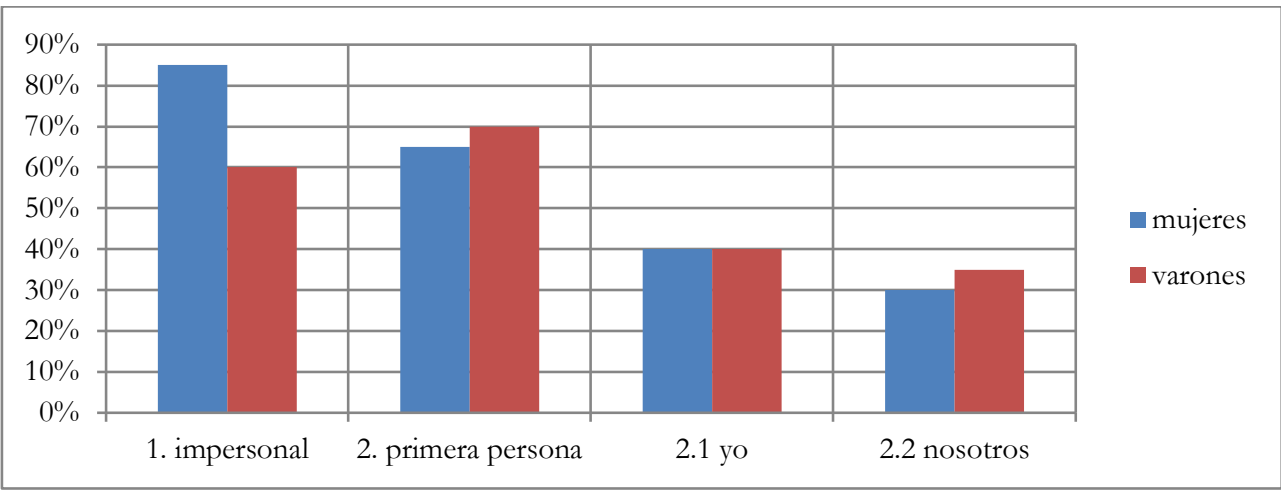

Gráfico 1. El sujeto verbal como enunciador-evaluador, según el sexo del evaluador.

En principio, la disparidad más acentuada se establece únicamente en el uso del impersonal por parte de varones $(60 \%)$ y mujeres $(85 \%)$. Esto muestra una diferencia significativa con un $90 \%$ de confianza (significancia de diferencia entre proporciones 
en un Test de Hipótesis). En ambos sexos prevalece en los ejemplos el verbo 'recomendar' (o 'sugerir'), esto es, las imposiciones de corrección enunciadas por la fuente impersonal evaluadora ('se') aparecen atenuadas, además, al presentarse como meras recomendaciones: 'se recomienda definir con más precisión'.

Se documentan otros verbos, también empleados por ambos sexos, en enunciados que constituyen una valoración negativa y atentan contra la imagen positiva de los autores; por ello, la responsabilidad de la emisión de la crítica se minimiza con el uso de la impersonalidad: 'se observa un desajuste general', 'se echa de menos', 'se señalan errores', 'se señalan carencias', ‘se marcan erratas', 'se advierte una desproporción', etc.

La recurrencia de aparición de la primera persona (en singular y/o en plural), en función del sexo, es muy similar: 65\% mujeres, 70\% varones. Es más, el empleo de la primera persona del singular 'yo' coincide en un 40\% en ambos sexos. En cambio, se observan diferencias respecto al lexema utilizado, ya que las mujeres optan sobre todo por el verbo doxástico 'creo' para introducir valoraciones negativas y por el verbo no impositivo, 'sugiero' ('anoto sugerencias', 'hago sugerencias'). Evaluadores de ambos sexos utilizan el verbo de carácter menos subjetivo, 'considero', y el verbo no impositivo 'recomiendo'. También evaluadores y evaluadoras recurren a otros verbos para establecer acciones más concretas y formular críticas ('cambio el título', 'separaría los párrafos', 'no entiendo el objetivo', 'cuestiono el uso de', 'he observado', 'anoto', 'cuestiono', 'detallaré cuestiones', 'no renunciaría a explicar más', 'cambiaría el enfoque', 'ofrezco ejemplos de actividad', etc.).

En el caso de la primera persona del plural 'nosotros', sucede lo mismo, ya que la cuantificación es muy similar (las mujeres, 30\% y los varones, 35\%). Sin embargo, de nuevo las diferencias surgen en relación con el lexema verbal empleado. Las mujeres optan mayoritariamente por no imponer una actuación en el autor a través de la recomendación ('recomendamos', 'sugerimos', 'animamos', etc.); por el contrario, ambos sexos emplean diferentes verbos que expresan la crítica o la imposición como producto de un proceso de cognición ('entendemos', 'somos conscientes de', 'no vemos su uso real', 'no sabemos la utilidad', 'incidimos en los siguientes puntos', etc.).

Respecto a la figura del autor como sujeto del enunciado, se aprecia una clara estrategia de impersonalidad, esto es, se emiten unas directrices que deben ser asumidas por un destinatario apenas presente en el texto: el autor apenas consta en el informe como un sujeto al que se dirige directamente el mensaje. Aparece sustituido por la impersonalidad y por el propio 'artículo' como sujeto del enunciado, en especial, en el caso de los varones, tal y como se observa en la Gráfico 2. 


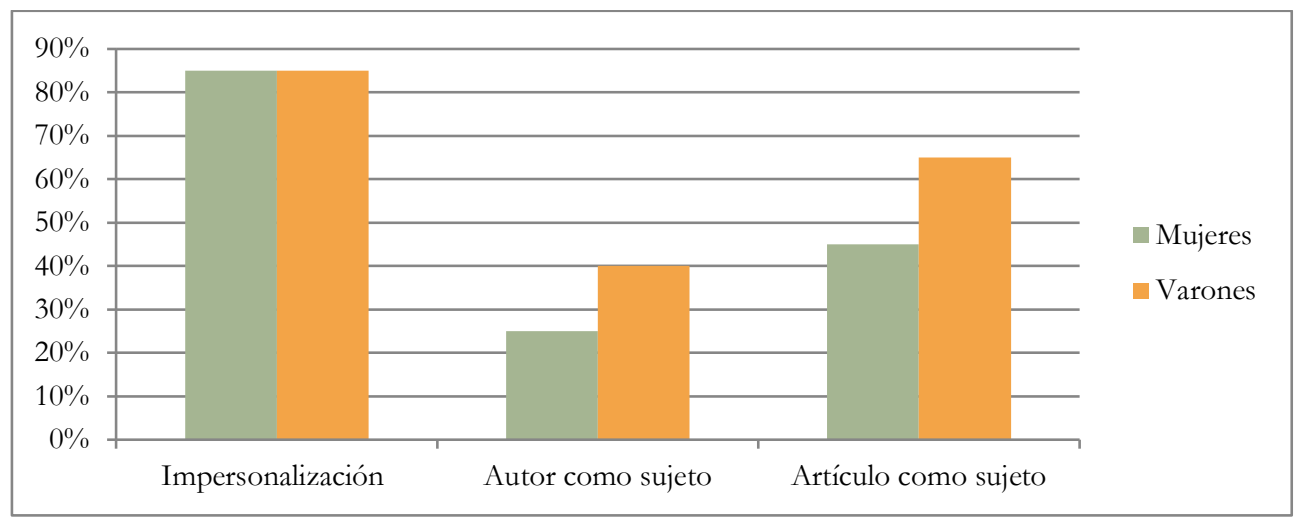

Gráfico 2. El sujeto verbal como autor del artículo, según el sexo del evaluador.

Esta estrategia de impersonalidad respecto al autor obedece al carácter de destinatario indirecto, es decir, el evaluador juzga un artículo y describe lo que debe modificarse, pero su interlocutor no es el autor; en el fondo, se dirige al equipo editorial como mediador que debe transmitir al autor sus observaciones. Esta estrategia de impersonalidad del destinatario se vincula a acciones impositivas que (para el autor) son obligatorias, especialmente con el verbo 'deber' ('se deberían desarrollar', 'deben introducirse'), o como acciones de modificación opcionales o aconsejables ('sería conveniente hacer una comparativa', 'sería preferible', 'convendría hacer una reestructuración profunda', 'podría reorganizarse', 'podría incluirse', 'podría añadirse'). En ambos casos, las modificaciones obligatorias o presentadas como opcionales (aunque, en realidad, no lo son) pueden ir acompañadas del condicional de cortesía. La única diferencia que se observa en relación con sexo tiene que ver de nuevo con el tipo de verbos y la acción verbal: en el caso de los evaluadores varones aparecen verbos en actos de crítica ('no se refleja', 'no se aportan casi referencias') y en el caso de las evaluadoras no se aprecian estas críticas impersonales, al sustituirse por una propuesta de mejora: 'sería oportuno', 'sería bueno', 'sería útil', 'sería interesante', 'sería deseable', etc.

Cuando el autor aparece como sujeto verbal siempre responde a una imposición de actuación real o presentada como opcional, en general, atenuada por el recurso al condicional: 'el autor debería apoyar sus teorías' o 'el autor podría ampliar la explicación'. Este tipo de acciones impositivas con apelación directa al autor aparecen más en varones evaluadores $(40 \%)$ que en mujeres $(25 \%)$, lo que implica una diferencia significativa.

Por último, cuando el propio artículo (o una parte) aparece como sujeto verbal suele utilizarse en actos de crítica: 'el trabajo adolece', 'la propuesta no queda clara', 'el artículo adolece', 'los objetivos no quedan claros', 'el artículo presenta considerables carencias', etc.; y en algunos casos, el evaluador opta por solicitar una actuación: 'esta 
sección requiere', 'el artículo necesita una revisión’ Este tipo de críticas directas o imposiciones es menos frecuente en evaluadoras (45\%) que en evaluadores $(65 \%)$.

\section{Discusión}

Si se retoman las preguntas de la investigación, el análisis realizado confirma la existencia de cuatro tipos de mecanismos lingüísticos para atenuar las críticas al artículo o las imposiciones de actuación a su autor, una atenuación ya solicitada por las propias revistas a los evaluadores. Estos mecanismos permiten velar por la imagen del evaluador y del autor: se trata de autoproteger la imagen del evaluador y reparar la crítica o la imposición realizada al autor frente a la figura del editor, ya que es el único que conoce las verdaderas identidades de ambos.

El estudio también ha posibilitado comprobar cómo las diferentes estrategias de cortesía atenuadora se suelen superponer y, en concreto, el predominio de un recurso: el condicional como minimizador. En este sentido la descripción realizada en esta investigación coincide con los datos cuantificados por Suid et al. (2018): la estrategia más empleada con diferencia es la del condicional (38\%), seguida de empleo de verbos performativos de creencia y opinión (19\%), de los verbos de recomendación y sugerencia (15\%); y de las construcciones impersonales e indeterminadas (14\%), expresiones de duda (4\%).

En cuanto a si existe alguna correlación entre la minimización del sujeto del enunciado y el sexo de los evaluadores, la respuesta resulta más compleja. Desde la perspectiva cuantitativa, se aprecian ciertas diferencias significativas en relación con el recurso a la impersonalidad y, además, se contempla un estilo discursivo, vinculado con los verbos y los tipos de actos de habla realizados. Las evaluadoras optan especialmente por un estilo discursivo en el que prevalece la estrategia de recomendación tanto conectada a una impersonalidad enunciativa como a un plural de modestia: ‘se recomienda' o 'recomendamos'; y, además, la crítica o la imposición al autor se tamizan a partir de la subjetividad de un uso mayoritario de 'creo'. En cambio, los varones evaluadores optan por convertir el artículo en el sujeto directo de la crítica y de la actuación de modificación, y la recomendación es mucho menor al solicitar actuaciones concretas ‘propongo' o emitir críticas como 'no vemos'.

Quizá esto conectaría con esas estrategias diferentes que emplean en el discurso hombres y mujeres, tal y como apuntaba García Mouton (1999) o con las diferentes formas de atenuar en entrevistas, según señalaba Albelda (2018): la mitigación de la opinión por parte de la persona que habla en el caso de las mujeres ('creo'). Ahora bien, a diferencia de Cestero (2017) y Albelda (2018), la impersonalidad no es mayoritaria en varones y tampoco se puede afirmar que el recurso al paralenguaje es más frecuente en mujeres, dado que el informe es un texto escrito. Esto podría implicar que en cada género discursivo se activan diferentes estrategias de cortesía por 
parte de mujeres y varones. En este género en concreto la impersonalidad vinculada al sujeto evaluador es mayor en mujeres que en varones.

Para futuros estudios de correlación de variables sociales (sexo o adscripción cultural) del evaluador y empleo de una cortesía atenuadora en informes de evaluación o en otros géneros discursivos, se propone el diseño de un corpus controlado, en el que un único artículo sea juzgado por evaluadores de distintas variables sociales. Esto permitirá obtener unos resultados más comparables y controlar mejor las variables.

\section{CONCLUSIONES}

El análisis de una muestra de informes de arbitraje ha permitido comprobar cómo el género discursivo y su anclaje situacional condicionan el empleo de una cortesía atenuadora, en este caso, por el doble juego de destinatarios del informe: el editor de la revista y el autor del artículo. El editor-juez es el destinatario directo del informe y puede sopesar la imagen positiva del evaluador y, por ello, aunque considerado como autoridad en la materia y como sujeto desinteresado al evaluar de un modo altruista y objetivo, su discurso debe ser estratégicamente cortés en sus imposiciones respecto a las correcciones que debe llevar a cabo el autor o en su valoración del artículo.

Si hasta la fecha se ha tenido en cuenta el grado de formación del evaluador y la recomendación o no de publicación del artículo como parámetros que afectan a la configuración lingüística del informe, en esta investigación se ha comprobado que el sexo del evaluador no repercute solo en el empleo de la estrategia de impersonalidad desde una perspectiva cuantitativa, sino en la construcción de distintos estilos discursivos en mujeres y varones como conjunción de varias estrategias.

No obstante, quedan numerosos retos pendiente para estudios futuros, como ampliar la muestra de sujetos por sexo para corroborar esta conclusión inicial; incrementar los elementos contrastados teniendo en cuenta no solo el sexo de los evaluadores, sino también el campo de adscripción de la revista, y la procedencia geolectal/cultural de la lengua del evaluador; y revisar las diversas variables lingüísticas. Para ello, se propone diseñar un experimento controlado: evaluar diferentes sujetos un único artículo.

\section{REFERENCIAS BIBLIOGRÁFICAS}

Albelda, M. (2018). Variación sociolingüística de los mecanismos mitigadores: Diferencias de uso en edad y sexo. Cultura, lenguaje y representación, XIX, 7-29.

Albelda, M. \& Barros, Mª J. (2013). La cortesía en la comunicación. Madrid: Arco Libros.

Albelda, M., Briz, A., Cestero, A. M., Kotwica, D. \& Villalba, C. (2014). Ficha metodológica para el análisis pragmático de la atenuación en corpus discursivos del español (ES.POR.ATENUACIÓN). Oralia, 17, 7-62. 
Askehave, I. \& Swales, J. M. (2001). Genre identification and communicative purpose: A problem and a possible solution. Applied Linguistics, 222, 195-212.

Astudillo, C., Squadrito, K., Varas, G., González, C. \& Sabaj, O. (2016). Polaridad de los comentarios y consistencia interna en los informes de arbitraje de artículos de investigación. Acta Bioethica, 22(1), 119-128.

Bhatia, V. K. (2002). Applied genre analysis: A multi-perspective model. Ibérica, 4, 319.

Bolívar, A. (2008). El informe de arbitraje como género discursivo en la dinámica de la investigación. Revista Latinoamericana de Estudios del Discurso, 8(1), 41-64.

Bolívar, A. (2011). Funciones discursivas de la evaluación negativa en informes de arbitraje de artículos de investigación en educación. Núcleo, 28, 59-89.

Bolívar Orellana, A. C. (2014). El informe de arbitraje: Datos para su estudio. Paradigma, 35(2) 213-234.

Bravo, D. \& Briz, A. (2004) (Coords.). Pragmática sociocultural: Estudios sobre el discurso de cortesía en español. Barcelona: Ariel Lingüística.

Brown, P. \& Levinson, S. C. (1987). Politeness: Some universals in language usage. Cambridge: Cambridge University Press.

Cestero, A. M. (2017). La atenuación en el habla de Madrid: Patrones sociopragmáticos. RILCE: Revista de Filología Hispáinca, 33(1), 57-86.

Fortanet, I. (2008). Evaluative language in peer review referee reports. Journal of English for Academic Purposes, 7, 27-37.

Fuentes, M. (2018). Patrones funcionales-valorativos en informes de arbitraje de artículos de investigación. Tesis doctoral, Pontificia Universidad Católica de Chile, Santiago, Chile.

García Mouton, P. (1999). Cómo hablan las mujeres. Madrid: Arco Libros.

Gosden, H. (2003). 'Why not give us the full story?': Functions of referees' comments in peer reviews of scientific research papers. Journal of English for Academic Purposes, 2, 87-10.

Haverkate, H. (1994). La cortesía verbal. Estudio pragmalingüístico. Madrid: Gredos.

Kourilova, M. (1998). Communicative characteristics of reviews of scientific papers written by non-native users of English. Endocrine Regulations, 32, 107-114. 
Mungra, P. \& Webber, P. (2010). Peer review process in medical research publication: Language and content comment. Journal of English for Specific Purposes, 29, 4353.

Paltridge, B. (2015). Referees' comments on submissions to peer-reviewed journals: When is a suggestion not a suggestion? Studies in Higher Education, 40(1), 106122.

Parodi, G. (2009). Géneros discursivos y lengua escrita: Propuesta de una concepción integral desde una perspectiva sociocognitiva. Revista Letras, 51(80), 19-55.

Sabaj, O., González, C., Varas, G. \& Pina-Stranger, A. (2015). A new form for the evaluation of scientific articles under peer review. Revista Argos, 32(62), 119129.

Sabaj, O., González, C., Astudillo, C., Varas, G., Fuentes, M., Cabezas, P., Squadrito, K. \& Pina-Strange, A. (2018). El informe de arbitraje según la recomendación de publicación y la productividad de los evaluadores. Athenea Digital, 18(2), 1 26.

Samraj, B. (2016). Discourse structure and variation in manuscript review. Implications for genre categorization. English for Specific Purposes, 42, 76-88.

Soler Bonafont, A. (2016). La función atenuante de los verbos doxásticos en español. Revista Internacional de Lingüistica Iberoamericana, XIV-1(27), 75-90.

Suid, N. (2016). Cortesía atenuadora en informes de arbitraje: Una mirada sociodiscursiva al proceso de evaluación por pares. Tesis de Magíster, Pontifica Universidad Católica de Chile, Santiago, Chile.

Suid, N., Sabaj, O. \& González, C. (2018). Ciencia, estatus y cortesía: Atenuación en informes de arbitraje de artículos de investigación. Tonos digital, 34, 1-27.

Swales, J. M. (1996). Occluded genres in the academy: The case of the submission letter. En E. Ventola \& A. Mauranen (Eds.), Academic writing: Intercultural and textual issues (pp. 45-58). Ámsterdam: John Benjamins.

Varas, G. (2015). El informe de arbitraje en el proceso de revisión por pares de artículos de investigación: Niveles de retroalimentación según el tipo de evaluador. Tesis de Magíster, Universidad de La Serena, La Serena, Chile. 


\section{NOTAS}

${ }^{1}$ Esta investigación se enmarca en el proyecto La atenuación pragmática en su variación genérica: géneros discursivos escritos y orales en el español de España y América (Ministerio de Economía y Competitividad de España, ref. FFI2016-75249-P).

2 Se entiende que la 'relación funcional' se deriva de la identidad y papel que se adquiere en cada evento comunicativo (profesor-estudiante o médico-paciente) y de la jerarquía en el proceso comunicativo (gestión del turno). Para Fuentes (2018: 20), se trataría de cierta relación asimétrica: "Otra variable que influye en la caracterización de los actores son las relaciones de poder entre evaluadores y autores. De esta forma, se establece que la relación entre autores y evaluadores es entre pares, entre colegas. A pesar de este hecho, hay una evidente superioridad de poder por parte del evaluador sobre los autores."

${ }^{3}$ Para Albelda et al. (2014), la atenuación cumple cuatro funciones: (a) reducir el compromiso del hablante con lo dicho, sin vinculación directa con la imagen; (b) autoprotegerse por lo dicho o por lo hecho, en relación con la imagen; (c) prevenir de una amenaza a la imagen del otro y (d) reparar de una amenaza a la imagen del otro.

${ }^{4}$ Los aspectos que atentan contra la 'imagen positiva' (adjetivos evaluativos negativos o críticas por la ausencia de elementos como 'falta' o 'no hay'...) o 'imagen negativa' (verbos en imperativo o perífrasis de obligación, se observan especialmente con elementos relativos a la expresión formal o ajustes a las normas editoriales, y en relación con la metodología.

5 Es lo que Soler Bonafont (2016: 3) considera como función atenuante de estos verbos doxásticos: "Con su uso, el emisor se desresponsabiliza de lo dicho y sitúa su enunciado en el terreno de la opinión y no en el de lo factual (Mullan, 2010), porque el hablante no está seguro de lo afirmado o finge no estarlo con el fin de no violar la máxima conversacional de cualidad (Aijmer 1997: 8)". Para su estudio la autora tiene en cuenta la explicitud del sujeto, su integración oracional y su posición discursiva. En nuestro caso, los sujetos siempre están implícitos y el verbo tiene una función oracional de verbo principal frente al posible uso parentético cercano al marcado discursivo gramaticalizado. 